\title{
CARTOGRAFÍA DE ZONAS PROBABLES DE SALIDA DE ALUDES EN EL ALTO GÁLLEGO (PIRINEO CENTRAL ARAGONÉS) MEDIANTE EL EMPLEO DE SISTEMAS DE INFORMACIÓN GEOGRÁFICA
}

\author{
J. CHUECA CÍA ${ }^{(1)}$ y A. JULIÁN ANDRÉS ${ }^{(2)}$ \\ (1) Dpto. de Geografía y Ordenación del Territorio \\ Facultad de Ciencias Humanas y de la Educación \\ Universidad de Zaragoza; 22002 Huesca \\ (2) Dpto. de Geografía y Ordenación del Territorio \\ Escuela Politécnica Superior \\ Universidad de Zaragoza; 22071 Huesca
}

Correo electrónico de contacto: jchueca@unizar.es

\begin{abstract}
RESUMEN. En el presente trabajo se realiza una cartografía de zonas probables de salida de aludes en el valle del Alto Gállego (Pirineo Central aragonés) a partir del empleo de Sistemas de Información Geográfica (ArcGIS). Los factores condicionantes utilizados para generar el modelo general de zonas probables de salida de aludes (MGZPSA) fueron: altitud mensual de la isoterma regional de $0^{\circ} \mathrm{C}$ (diciembre-abril), pendientes y cubierta del terreno. La incorporación adicional de la variable de radiación solar mensual permitió clasificar estas zonas en tres grados de susceptibilidad al desencadenamiento de aludes en función de los valores de radiación registrados en las mismas y su contribución a la inestabilidad del manto nival (modelo radiación solar; MRSZPSA). Los dos modelos se validaron utilizando dos áreas de observación de orientaciones contrastadas localizadas en la parte alta del valle.
\end{abstract}

ABSTRACT. We present in this work the cartography of probable avalanche starting zones in the Alto Gállego valley (Spanish central Pyrenees) made by using a Geographical Information System (ArcGIS). The conditioning factors used to generate the general model for probable avalanche starting zones (MGZPSA) were: altitude of the monthly regional $0^{\circ} \mathrm{C}$ isotherm (DecemberApril), slope and surface cover. The additional incorporation of the monthly solar radiation variable allowed to classify these areas into three susceptibility sectors according to the radiation inputs and their subsequent contribution to the instability of the snowpack (solar radiation model; MRSZPSA). The two models 
were validated using two control areas with contrasted aspect located in the high sector of the valley.

Palabras clave: cartografía, zonas probables salida aludes, Sistemas Información Geográfica, Alto Gállego, Pirineo Central.

Key words: cartography, avalanche starting zones, Geographical Information Systems, Alto Gállego, Central Pyrenees.

Enviado el 6 de noviembre de 2009 Aceptado el 9 de enero de 2010

\section{Introducción}

Entre los riesgos naturales que afectan a las áreas de montaña, el desencadenamiento de aludes ocupa un lugar significativo. En Aragón, los aludes sólo se presentan de forma habitual en la Cordillera Pirenaica, de forma que es en esta cadena montañosa en la que se ha llevado a cabo la mayor parte de los trabajos relacionados con aspectos teóricos y prácticos de este proceso geomorfológico. El Pirineo aragonés, el tramo de la cadena pirenaica con mayores elevaciones y más escarpada topografía, cuenta hasta la fecha con varios trabajos de detalle en los que se han cartografiado zonas de riesgo o caracterizado la tipología de aludes (López Guarga et al., 1997; Julián y Chueca, 1999, 2008; Julián et al., 2001; Chueca y Julián, 2004).

Uno de los sectores analizados más recientemente (Palomo, 2007; Palomo et al., 2008; Chueca et al., 2009), representativo tanto por la ubicuidad de las avalanchas como por tratarse de un territorio en el que las actividades recreativas ligadas a la montaña alcanzan una gran relevancia, es el valle del río Aguas Limpias (valle de Tena; Pirineo central aragonés). En el presente trabajo se realiza, mediante el empleo de Sistemas de Información Geográfica, la cartografía escala 1:25.000 de zonas probables de salida de aludes de un sector próximo, el alto valle del río Gállego, en concreto la zona localizada aguas arriba de la localidad de Sallent de Gállego. Las vertientes que modelan este tramo del Gállego, por su altitud, pendientes y grado de cubierta vegetal, son muy favorables al desarrollo de aludes de nieve, y el estudio de fuentes documentales confirma la recurrencia en el sector de este tipo de procesos desde hace décadas.

Nuestra investigación toma como referencia metodológica la cartografía de zonas probables de salida de aludes efectuada para una parte del valle de Ordesa (Julián y Chueca, 2008), aunque se matizan y modifican varios de los parámetros de medición allí empleados y se introduce la validación de resultados. Los factores condicionantes utilizados para generar el denominado modelo general de zonas probables de salidas de aludes (MGZPSA) son: la altitud mensual de la isoterma regional de $0^{\circ} \mathrm{C}$ (diciembre-abril), las pendientes y la cubierta del terreno. La incorporación adicional de la variable de radiación solar mensual permite clasificar esas zonas en tres grados de susceptibilidad al desencadenamiento de aludes en función de los valores de radiación registrados en las mismas y su contribución a la inestabilidad del manto nival (modelo radiación solar; 
MRSZPSA). Los dos modelos se validan utilizando dos áreas de observación localizadas en la parte más alta del valle, con pendientes y orientaciones (radiación solar) contrastadas y un rango altitudinal suficientemente representativo.

\section{2. Área de estudio}

El área de estudio incluye el tramo de la cuenca del río Gállego localizado aguas arriba de la localidad de Sallent de Gállego (Fig. 1). Ocupa una extensión de 44,26 km² y presenta un contraste topográfico marcado, oscilando las alturas s.n.m. entre los 1.300 m en el cauce del río Gállego, y los más de $2.500 \mathrm{~m}$ en algunas de sus cumbres más elevadas del margen septentrional (Pic de l'Ouradé, $2.599 \mathrm{~m}$ ) y meridional (Pico Culivillas, 2.529 m; Pico Arroyeras, 2.551 m; Vértice de Anayet, 2.559 m).

En términos morfoestructurales, el alto Gállego se engloba dentro del Pirineo axial. La zona estudiada está compuesta en su totalidad por materiales paleozoicos. El distinto comportamiento de la litología ante la acción erosiva fluvial y glaciar se tradujo en dos tipos de modelados bien diferenciados: en las extensas áreas en las que dominan las pizaras aparecen divisorias redondeadas e importantes depresiones muy afectadas por grandes movimientos en masa (zona de Sallent o tramo Formigal-El Portalet); por el contrario, en

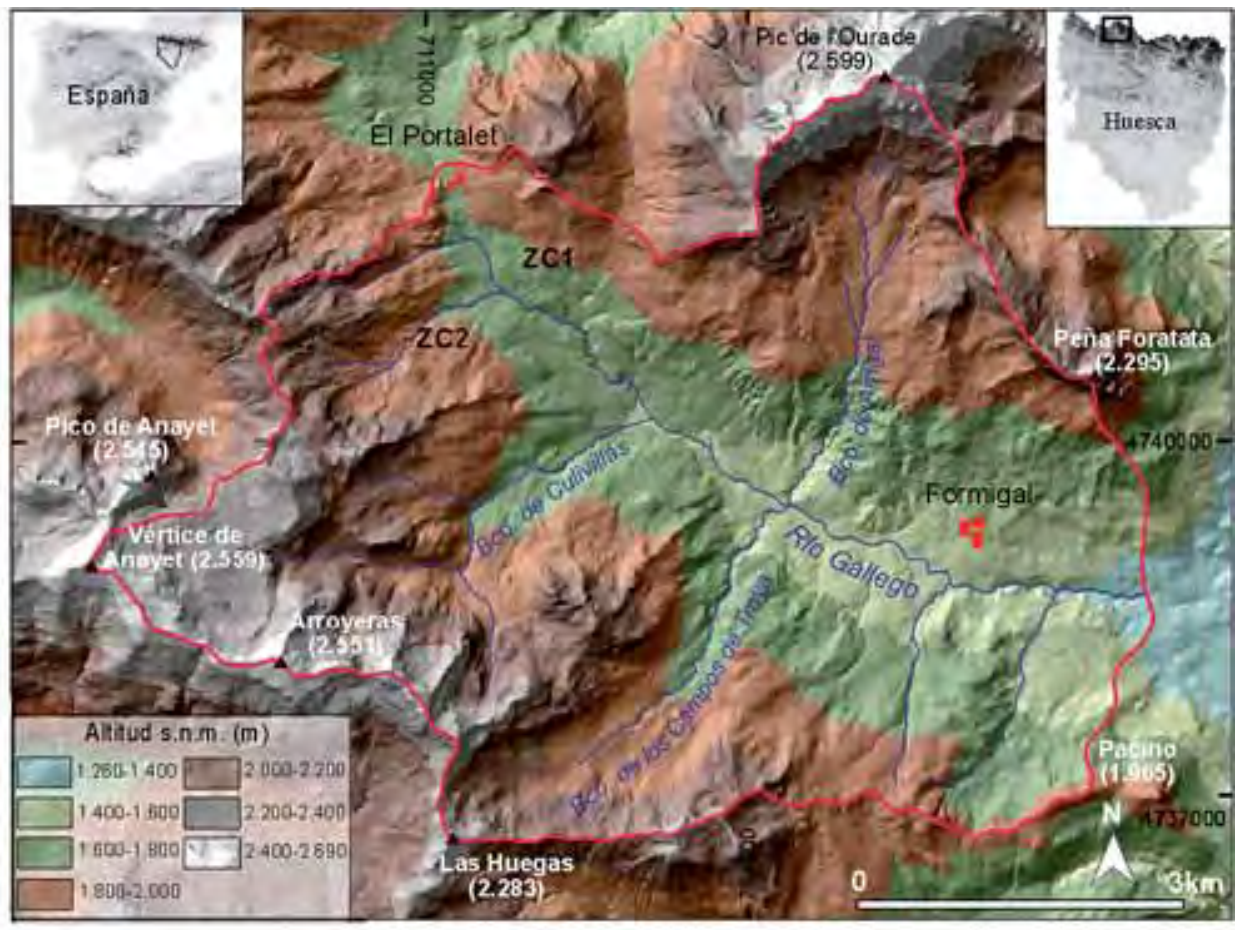

Figura 1. Localización del área de estudio (ZC1: zona de control 1; ZC2: zona de control 2). 







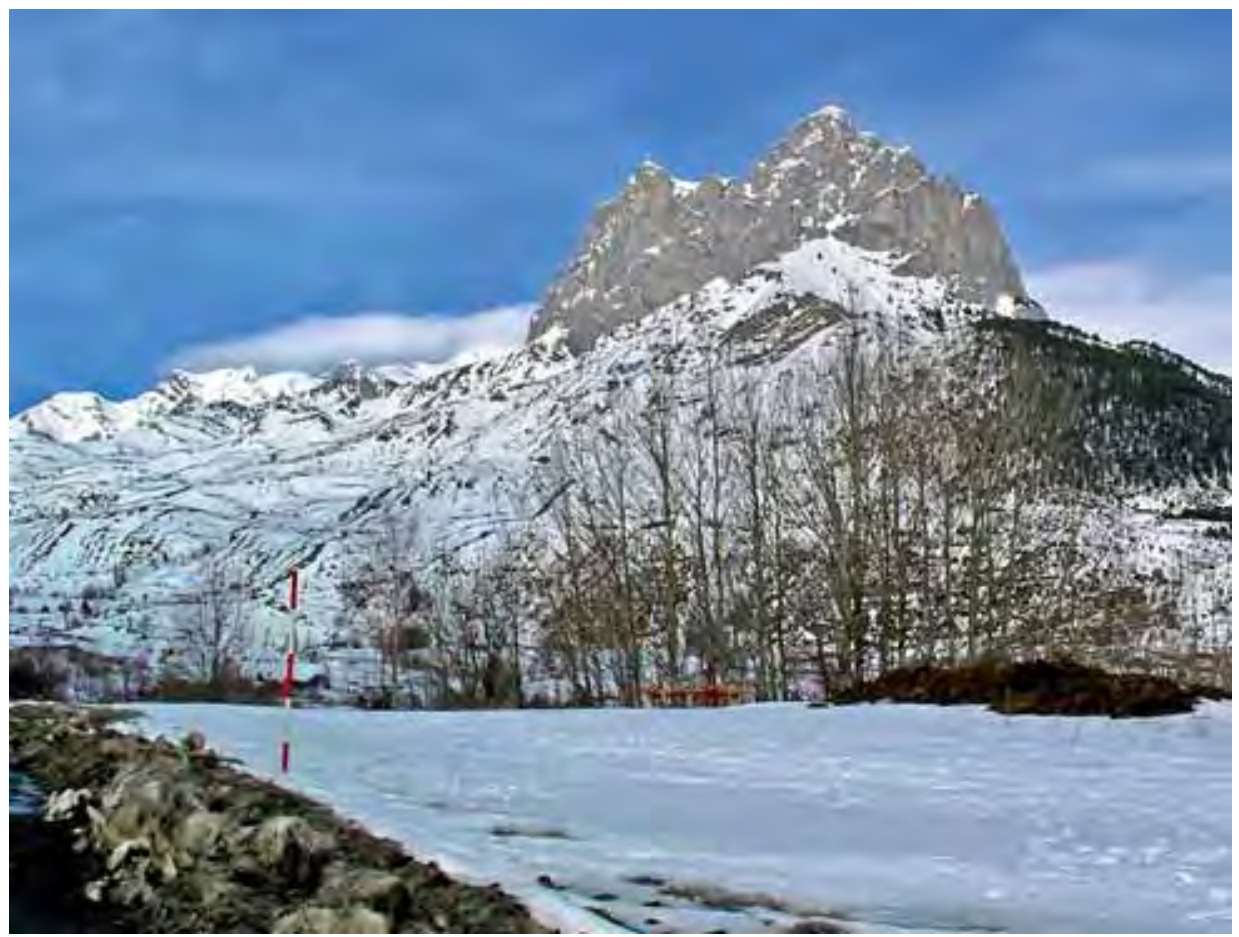

Figura 3. Panorámica del tramo inferior del área de estudio (sector Peña Foratata).

los sectores en los que afloran las calizas, éstas quedan en resalte conformando crestas y frentes de cuesta escarpados puntualmente afectados por procesos de karstificación (Pacino, Peña Foratata, Aneu-El Portalet). Adicionalmente, el afloramiento de materiales volcánicos de gran resistencia y masividad (andesitas, fundamentalmente) da lugar a la aparición de enérgicos relieves bien representados en la zona de Anayet, Espelunciecha, Arroyeras y Campo de Troya (Fig. 2) (Chueca et al., 2000; Julián et al., 2000).

El sector presenta un clima húmedo de montaña con influencia atlántica. Las precipitaciones muestran máximos en primavera y otoño, registrándose las nevadas más importantes entre diciembre y marzo; la altitud de la isoterma de $0^{\circ} \mathrm{C}$, que condiciona los fenómenos de innivación y permanencia de la nieve, exhibe una considerable variación anual (octubre, 2.998 m s.n.m; noviembre, $2.580 \mathrm{~m}$; diciembre, $1.917 \mathrm{~m}$; enero, $1.663 \mathrm{~m}$; febrero, $1.611 \mathrm{~m}$; marzo, $1.850 \mathrm{~m}$; abril, $2.091 \mathrm{~m}$; mayo, $2.569 \mathrm{~m}$ ), con un valor promedio para la estación de 1.708 m s.n.m. (de la Riva, 2000) (Fig. 3).

La cubierta vegetal de la zona es muy poco variada, dominando claramente la roca desnuda y los pastizales de montaña de los pisos alpino y subalpino. En superficies muy reducidas, localizadas principalmente al pie de la alineación Pico de los Tres HombresAnayet, aparecen distintas formaciones de matorral (gayuba, enebros rastreros, rodo- 
dendros). Existen algunas manchas aisladas de bosque abierto de pino negro en el sector ubicado al norte del Pico de Campo de Troya, aunque el único tramo con una cubierta forestal densa se localiza en el límite suroriental del área de estudio e incluye los bosques de hayas de La Tosquera-Pacino (Ibarra y Pérez Cabello, 2000).

\section{Material y métodos}

Como ya hemos indicado, el presente trabajo toma como referencia metodológica inicial la cartografía de zonas probables de salida de aludes canalizados de dinamismo medio efectuada recientemente para un sector del valle de Ordesa (Julián y Chueca, 2008). Las variables consideradas como básicas incluyen: 1) la altitud mensual de la isoterma regional de $0^{\circ} \mathrm{C}$. Marca la cota inferior a partir de la cual las precipitaciones se producen en forma de nieve y las bajas temperaturas favorecen su conservación; 2) las pendientes. Los aludes se originan en zonas con pendientes que oscilan entre $\operatorname{los} 25^{\circ} \mathrm{y}$ los $45^{\circ}$. Por debajo de $25^{\circ}$ el manto de nieve permanece estático a no ser que se produzcan situaciones atmosféricas inusuales que den lugar a nevadas extraordinarias. Por encima de los $45^{\circ}$ la nieve caída no se ancla a la cubierta del terreno o al manto subyacente, depositándose en niveles inferiores; y 3) la cubierta del terreno. Importante por sus repercusiones en la rugosidad del terreno, que a su vez limita (sustrato rugoso) o favorece (sustrato no-rugoso) el arranque de las avalanchas de nieve. En esta variable se engloban factores litológicos, geomorfológicos y la cubierta vegetal.

Como variable adicional se utilizó la 4) radiación solar. La radiación solar condiciona la temperatura de la superficie del terreno y, por consiguiente, altera la evolución del manto de nieve y su metamorfosis. La incorporación de este parámetro permite clasificar zonas atendiendo a su susceptibilidad potencial al desencadenamiento de aludes en función de los valores de radiación solar registrados en las mismas y su contribución a la inestabilidad del manto nival.

La información utilizada para analizar las variables mencionadas procede de varias fuentes, que se trabajaron en el contexto del Sistema de Información Geográfica ArcGIS:

1) Altimetría digital a escala 1:25.000 (Instituto Geográfico Nacional; Hojas 145I: Formigal y 145-II: Sallent de Gállego). A partir de ella se elaboró un Modelo Digital de Elevaciones (MDE) mediante la interpolación de las curvas de nivel y de las cotas, primero en formato TIN y, posteriormente, en formato raster con una resolución de malla de $10 \mathrm{~m}$. De este MDE se obtuvo: a) la cartografía derivada de pendientes favorables (valores comprendidos entre $\operatorname{los} 25^{\circ} \mathrm{y}$ $\left.45^{\circ}\right)$; y b) la cartografía con los valores mensuales de radiación solar. En este caso se trabajó con el módulo INSOLDIA del SIG MiraMon, que permite obtener para cada celda del Modelo Digital de Elevaciones la radiación solar potencial (medida en $10 \mathrm{KJ} \cdot \mathrm{m}^{-2} \cdot$ día $^{-1} \cdot \mu \mathrm{m}^{-1}$ ). En el proceso de cálculo, el programa considera la latitud, altitud, orientación, efecto de las sombras por la topografía circundante (precisión en acimut: $10^{\circ}$; precisión en altura: $1^{\circ}$ ), trayectoria solar a lo largo del día (horaria) y ángulos de incidencia en cada celda (precisión 
dependiente de la resolución planimétrica del MDE), distancia Tierra-Sol (mensual) y el efecto de extinción atmosférica (a falta de estaciones meteorológicas próximas, se utilizó el valor estándar recomendado de condiciones de atmósfera clara). Los intervalos de radiación solar que hemos considerado en el presente trabajo para determinar el grado de susceptibilidad potencial a la desestabilización de la nieve, y por tanto, al desencadenamiento de aludes, fueron los siguientes: susceptibilidad potencial baja: $94-1.01210 \mathrm{KJ} \cdot \mathrm{m}^{-2} \cdot \mathrm{dí}^{-1} \cdot \mu \mathrm{m}^{-1}$; susceptibilidad potencial media: $1.012-1.93010 \mathrm{KJ} \cdot \mathrm{m}^{-2} \cdot \mathrm{dí}^{-1} \cdot \mu \mathrm{m}^{-1}$; y susceptibilidad potencial alta: $1.930-2.84910 \mathrm{KJ} \cdot \mathrm{m}^{-2} \cdot \mathrm{día}^{-1} \cdot \mu \mathrm{m}^{-1}$. En la creación de estos tres intervalos se tuvieron en cuenta los valores de radiación solar mínima y máxima calculados a escala mensual para la zona de estudio (diciembre: 94 $10 \mathrm{KJ} \cdot \mathrm{m}^{-2} \cdot$ día $^{-1} \cdot \mu \mathrm{m}^{-1}$; abril: $\left.2.84910 \mathrm{KJ} \cdot \mathrm{m}^{-2} \cdot \mathrm{día}^{-1} \cdot \mu \mathrm{m}^{-1}\right)$.

2) Ortofotogramas del Plan Nacional de Ortofotografía Aérea (PNOA) del año 2006 (resolución de píxel de $50 \mathrm{~cm}$; color). A partir de su fotointerpretación se elaboró la cartografía de cubierta del terreno, identificándose las categorías de cubiertas favorables (roca desnuda, canchales, pastizales) y desfavorables (láminas de agua, bosque abierto, bosque denso) al arranque de aludes.

3) Información climática regional, procedente de la caracterización de la zona efectuada por de la Riva (2000) y utilizada para obtener la localización altitudinal mensual de la isoterma de $0^{\circ} \mathrm{C}$ en el área de estudio durante el período invernal (diciembre, $1.917 \mathrm{~m}$; enero, $1.663 \mathrm{~m}$; febrero, $1.611 \mathrm{~m}$; marzo, $1.850 \mathrm{~m}$; abril, $2.091 \mathrm{~m})$.

A las variables consideradas, una vez reclasificadas, se les aplicó una evaluación multicriterio de tipo booleano, obteniéndose dos modelos de delimitación de zonas de arranque de aludes a escala mensual: 1) el modelo general de zonas probables de salidas de aludes (MGZPSA) se basó en la combinación/superposición de los tres criterios básicos ya mencionados (altitud mensual de la isoterma regional de $0^{\circ} \mathrm{C}$, pendientes y cubierta del terreno); y 2) el modelo de susceptibilidad potencial en función de la radiación solar de zonas probables de salidas de aludes (MRSZPSA) se obtuvo añadiendo al anterior la variable radiación solar.

Los dos modelos se validaron utilizando dos áreas de observación localizadas en la parte más alta del valle (zona de control 1: sector El Portalet-Puerto Viejo de Sallent; zona de control 2: valles de Bacarizal y Espelunciecha) (Fig. 1). Las dos zonas de control elegidas, con buena visibilidad desde puntos estratégicos de fácil acceso, incluyen cuencas fluviales de tamaño pequeño y medio con pendientes y orientaciones (radiación solar) contrastadas y un rango altitudinal suficientemente representativo. En ellas se han ido llevando a cabo, a lo largo de los últimos dos años, observaciones durante los meses invernales para confirmar el desencadenamiento de aludes en sus vertientes y su correspondencia con lo predicho en los modelos. Los aludes identificados se incluyen en los cuatro tipos básicos usualmente mencionados en la literatura [aludes de nieve suelta (seca / húmeda); aludes de placa (seca / húmeda)] (McClung y Schaerer, 1996). Esta validación no es sistemática, al no haberse efectuado un muestreo de tipo aleatorio 
repartido por toda la zona de análisis, pero es suficientemente indicativa de la fiabilidad de la cartografía efectuada. Un muestreo de tipo aleatorio hubiera implicado la observación de sectores con acceso complicado o difícil y la inclusión de numerosas vertientes sometidas artificialmente a procesos de purgas y aludes controlados incluidas en el dominio de la estación de esquí de Formigal, que abarca una buena parte de la zona meridional del área de estudio (en este sentido, las dos zonas de control elegidas presentan una antropización mucho menor o nula, al menos hasta la fecha).

La fiabilidad y significación de los modelos se evaluó a partir del estadístico Kappa y de medidas de calidad adicionales derivadas de la matriz de confusión (sensibilidad o precisión general e intervalos de confianza al 95\% asociados). El estadístico Kappa se utiliza para medir el acuerdo o concordancia entre dos conjuntos de observaciones, teniendo en cuenta la probabilidad de obtener acuerdos por azar. En nuestro caso, los dos conjuntos de datos a comparar en cada modelo fueron las observaciones de aludes confirmadas sobre el terreno en cada zona de control (total del período diciembre-abril) frente a las modelizadas. Los valores Kappa $(K)$ oscilan entre $\leq 0$ (acuerdo nulo) y 1 (acuerdo máximo) $(K<0,20=$ acuerdo pobre; $0,21<K<0,40=$ acuerdo débil; $0,41<$ $K<0,60=$ acuerdo moderado; $0,61<K<0,80=$ acuerdo bueno; $K>0,81=$ acuerdo muy bueno; Landis y Koch, 1977). La sensibilidad o precisión general de un modelo $(S)$ se define como el cociente entre el número de casos correctamente clasificados y el total de casos de la muestra.

\section{Resultados y discusión}

La cartografía elaborada (con una escala original 1:25.000) muestra las zonas probables de salida de aludes en el área de estudio a lo largo de la temporada invernal. No es, por tanto, una cartografía de zonas probables de aludes (Julián y Chueca, 1999; Julián et al., 2001; Palomo et al., 2008), en la que se delimitan, a partir de procesos adicionales de fotointerpretación, trabajo de campo y encuesta, todas las áreas susceptibles al desarrollo (arranque, trayecto y llegada) de aludes. El interés de la elaboración de la cartografía de zonas probables de salida de aludes radica en la facilidad de su construcción a partir de procedimientos automáticos integrados en el contexto de un Sistema de Información Geográfica. Por otro lado, además de situar de forma muy precisa los tramos de arranque de avalanchas, este tipo de mapas (trabajando a escalas de detalle como la utilizada en el presente trabajo) permite valorar de una forma aproximada pero suficientemente eficiente, gracias a la posibilidad de inserción de la información morfotopográfica facilitada por los MDE, los sectores potencialmente sujetos al paso y a la llegada de avalanchas, aunque esas zonas no lleguen a cartografiarse explícitamente.

Los resultados obtenidos se reflejan, condensados, en las Figuras 4 y 5 (como ya hemos mencionado, la cartografía original está elaborada a escala 1:25.000). En la Figura 4 aparecen cartografiadas, por meses, las zonas probables de salidas de aludes obtenidas a partir del modelo general (MGZPSA). Teniendo en cuenta la superficie total de la cuenca $\left(44,26 \mathrm{~km}^{2}\right)$, la extensión ocupada a lo largo del invierno por los sectores propensos al arranque de avalanchas es la siguiente: diciembre, 7,32 $\mathrm{km}^{2}$ 


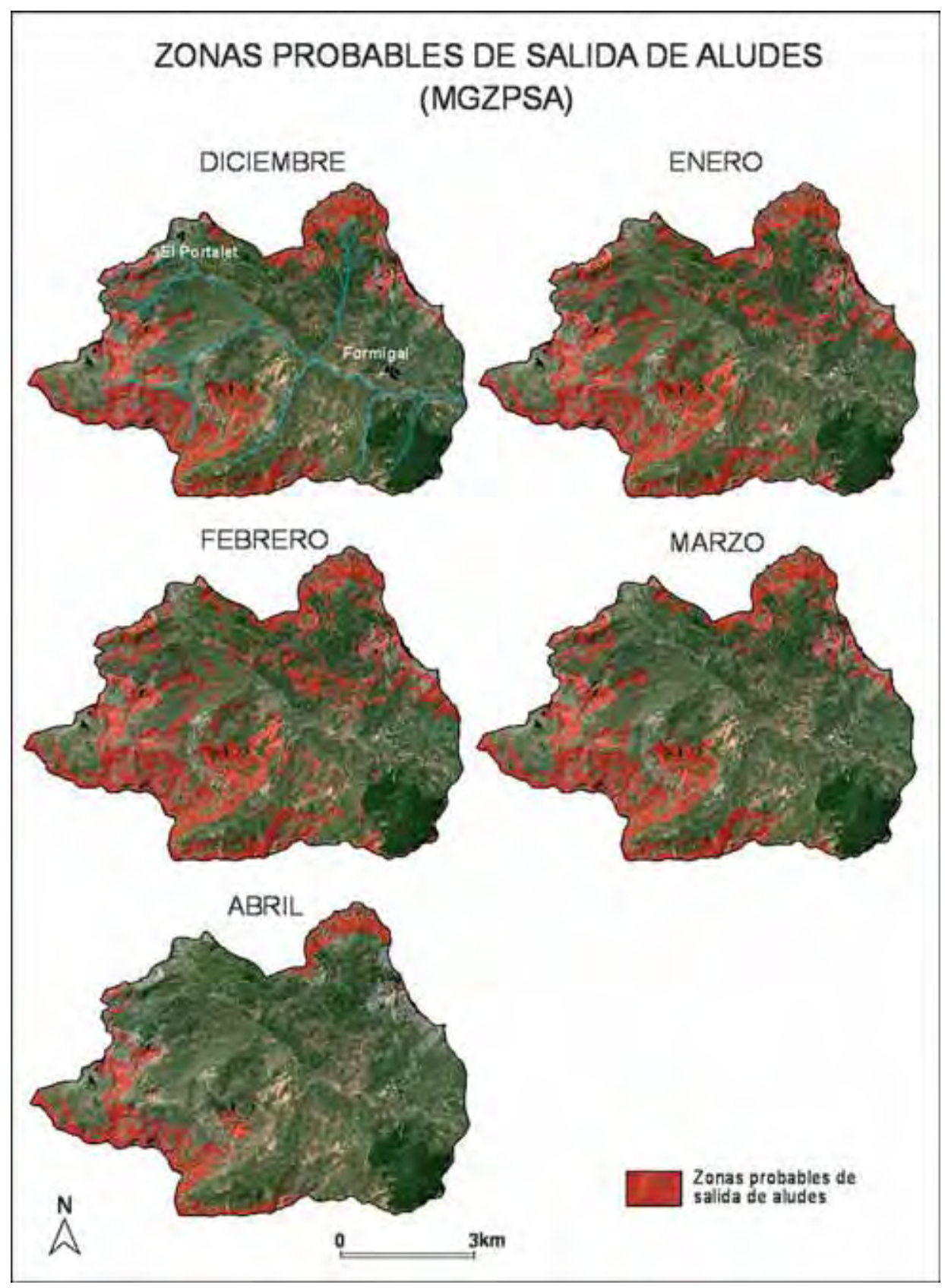

Figura 4. Modelo general de zonas probables de salidas de aludes (MGZPSA). 




Figura 5. Modelo de susceptibilidad potencial en función de la radiación solar de zonas probables de salidas de aludes (MRSZPSA). 
(16,53\%); enero, $10,61 \mathrm{~km}^{2}$ (23,97\%); febrero, $10,98 \mathrm{~km}^{2}$ (24,80\%); marzo, 8,34 km² $(18,84 \%)$; abril, 3,92 $\mathrm{km}^{2}(8,85 \%)$. En la Figura 5 se recogen las zonas probables de salidas de aludes obtenidas a partir del modelo de susceptibilidad potencial en función de la radiación solar (MRSZPSA). En este caso, como es lógico, la extensión espacial de los sectores propensos al arranque de avalanchas varía en función de la evolución anual de las entradas de radiación solar en las laderas, y es la siguiente: 1) Susceptibilidad potencial baja: diciembre, 5,93 $\mathrm{km}^{2}$ (13,39\%); enero, 6,95 $\mathrm{km}^{2}$ (15,70\%); febrero, 5,21 km² (11,77\%); marzo, 2,34 km² (5,28\%); abril, 0,01 km² (0,02\%); 2) Susceptibilidad potencial media: diciembre, $1,39 \mathrm{~km}^{2}(3,14 \%)$; enero, 3,65 $\mathrm{km}^{2}$ (8,24\%); febrero, 5,65 $\mathrm{km}^{2}$ (12,76\%); marzo, 2,66 km² (6,00\%); abril, 1,61 km $(3,63 \%) ; 3)$ Susceptibilidad potencial alta: febrero, $0,11 \mathrm{~km}^{2}(0,26 \%)$; marzo, 3,33 $\mathrm{km}^{2}(7,52 \%)$; abril, $2,30 \mathrm{~km}^{2}(5,19 \%)$.

En el mes de febrero, en el que la isoterma de $0^{\circ} \mathrm{C}$ se sitúa a una cota menor $(1.611$ m s.n.m.) y la extensión ocupada por las zonas con probables arranques de aludes es, por tanto, máxima, los tramos del valle alto del Gállego afectados incluyen grandes porciones de las distintas subcuencas que drenan el valle, tanto las de orientación NE (Bacarizal, Espelunciecha, Culivillas, Campo de Troya), como las de orientación SO (El Portalet, Brocuso, Arrigal). En el mes de abril, con la isoterma de $0^{\circ} \mathrm{C}$ emplazada ya a una considerable altitud (2.091 m s.n.m.), los sectores afectados por posible desencadenamiento de aludes se reducen drásticamente y quedan limitados a las vertientes más altas de la mayor parte de las subcuencas de orientación NE y a la del barranco de Arrigal, en las de orientación SO.

Conviene resaltar que los valores obtenidos son aproximaciones teóricas basadas en la probabilidad de que se registren precipitaciones en forma nival partiendo de la localización altitudinal de la isoterma de $0^{\circ} \mathrm{C}$; la nieve acumulada en meses previos puede seguir conservándose en algunas de las laderas analizadas hasta bien entrada la primavera (y desencadenarse aludes sobre ellas), o pueden producirse eventos puntuales de precipitaciones nivales intensas que cubran la totalidad del área de estudio. Por tanto, la cartografía mensual aportada tiene un valor orientativo informándonos, fundamentalmente, de las tendencias a esa escala temporal en el reparto espacial de las zonas de salida de avalanchas.

Tabla 1. Valores Kappa (K) y de sensibilidad / precisión general $(S)$ e intervalos de confianza (IC) asociados (al 95\%) para cada uno de los modelos en las dos zonas de control (ZC1 y ZC2).

\begin{tabular}{|c|c|c|c|c|c|c|c|c|c|c|c|c|}
\hline & \multicolumn{3}{|c|}{ MGZPSA } & \multicolumn{3}{|c|}{$\begin{array}{c}\text { MRSZPSA } \\
\text { (suscep. potencial baja) }\end{array}$} & \multicolumn{3}{|c|}{$\begin{array}{c}\text { MRSZPSA } \\
\text { (suscep. potencial media) }\end{array}$} & \multicolumn{3}{|c|}{$\begin{array}{c}\text { MRSZPSA } \\
\text { (suscep. potencial alta) }\end{array}$} \\
\hline & $K$ & $S$ & IC & K & $S$ & IC & $K$ & $S$ & IC & K & $S$ & IC \\
\hline $\mathrm{ZC1}$ & 0,5714 & 0,7857 & $\begin{array}{c}0,5708- \\
1,0007\end{array}$ & $-0,3334$ & 0,3333 & $\begin{array}{c}-0,2001- \\
0,8668\end{array}$ & 0,6666 & 0,8333 & $\begin{array}{c}0,5351- \\
1,1315\end{array}$ & 1,0000 & 1,0000 & $\begin{array}{r}1,0000- \\
1,0000\end{array}$ \\
\hline $\mathrm{ZC} 2$ & 0,7242 & 0,8621 & $\begin{array}{c}0,7366- \\
0,9876\end{array}$ & 0,4286 & 0,7143 & $\begin{array}{c}0,3796- \\
1,0489\end{array}$ & 0,7500 & 0,8750 & $\begin{array}{r}0,6458- \\
1,1042\end{array}$ & 0,8572 & 0,9286 & $\begin{array}{r}0,7937- \\
1,0635\end{array}$ \\
\hline
\end{tabular}


Los resultados generales de la validación de los modelos en cada una de las dos zonas de control (ZC1 y ZC2) se recogen en la Tabla 1. El modelo general de zonas probables de salidas de aludes (MGZPSA) muestra mejor concordancia entre aludes observados / modelizados en la ZC2 (25 aludes observados vs. 29 modelizados; $K=0,7242$, acuerdo bueno) que en la ZC1 (11 aludes observados vs. 14 modelizados; $K=0,5714$, acuerdo moderado). La medida de calidad adicional proporcionada por la sensibilidad o precisión general del modelo también ofrece igualmente mejores resultados para la ZC2 $(S=0,8621)$ que para la ZC1 $(S=0,7857)$.

En el caso del modelo de susceptibilidad potencial en función de la radiación solar de zonas probables de salidas de aludes (MRSZPSA), existen claras diferencias entre las tres categorías diferenciadas. El MRSZPSA con susceptibilidad potencial baja (radiación solar: 94-1.012 $10 \mathrm{KJ} \cdot \mathrm{m}^{-2} \cdot \mathrm{dí}^{-1} \cdot \mu^{-1}$ ) ofrece, de los tres casos considerados, las peores concordancias en ambas zonas de control (ZC1: 1 alud observado vs. 3 modelizados; $K=-0,3334$, acuerdo pobre; $S=0,3333$; ZC2: 5 aludes observados $v s$. 7 modelizados; $K=0,4286$, acuerdo moderado; $S=0,7143$ ). En posición intermedia, pero ya con valores de concordancia aceptables, se sitúa el MRSZPSA con susceptibilidad potencial media (radiación solar: 1.012-1.930 $\left.10 \mathrm{KJ} \cdot \mathrm{m}^{-2} \cdot \mathrm{dí}^{-1} \cdot \mu \mathrm{m}^{-1}\right)(\mathrm{ZC} 1: 5$ aludes observados $v s$. 6 modelizados; $K=0,6666$, acuerdo bueno; $S=0,8333$; ZC2: 7 aludes observados vs. 8 modelizados; $K=0,7500$, acuerdo bueno; $S=0,8750$ ). Los mejores datos los ofrece, sin duda, y de nuevo para ambas zonas de control, el MRSZPSA con susceptibilidad potencial alta (radiación solar: 1.930-2.849 $\left.10 \mathrm{KJ} \cdot \mathrm{m}^{-2} \cdot \mathrm{dí}^{-1} \cdot \mu \mathrm{m}^{-1}\right)(\mathrm{ZC} 1: 5$ aludes observados vs. 5 modelizados; $K=1,0000$, acuerdo muy bueno; $S=1,0000 ; \mathrm{ZC} 2$ : 13 aludes observados vs. 14 modelizados; $K=0,8572$, acuerdo muy bueno; $S=0,9286$ ).

A la vista de estos resultados es apreciable, en general, la mayor calidad de los modelos en las zonas con predominio de orientaciones de componente NE (ZC2), frente a las zonas con predominio de orientaciones SO (ZC1) (Figs. 6 y 7). Dado que los factores condicionantes básicos pendientes y cubierta del terreno tienen un reparto homogéneo por la zona de estudio, es este factor orientación -cuantificado en nuestro caso a través de los valores de la radiación solar incidente- el que parece ayudar a matizar con mayor precisión el reparto espacial de las zonas de arranque de avalanchas.

Del examen de la calidad de los modelos MRSZPSA a lo largo de la temporada invernal se deduce una mayor capacidad predictiva en los meses en los que la radiación solar alcanza cantidades más elevadas (marzo, abril), frente a los meses con entradas de radiación solar menores (diciembre, enero) (esta tendencia es observable en las dos zonas de control, aunque los mejores resultados -en general- se aprecian en la ZC2). El hecho de que el mayor número de concordancias entre eventos observados / modelizados se produzca en los meses de marzo y abril, podría relacionarse con la especial incidencia que en estas fechas tienen los aludes de nieve suelta húmeda, que en su desarrollo dependen de una transformación del manto nivoso en la que los inputs de radiación solar juegan un papel fundamental. En los meses más fríos, la radiación solar repercute de forma menos notable en el desencadenamiento de las avalanchas observadas (fundamentalmente aludes de nieve suelta seca y aludes de placa seca), aunque parece que lo haría de modo algo más marcado en las vertientes con orientaciones de componente norte que en las de componente meridional. 


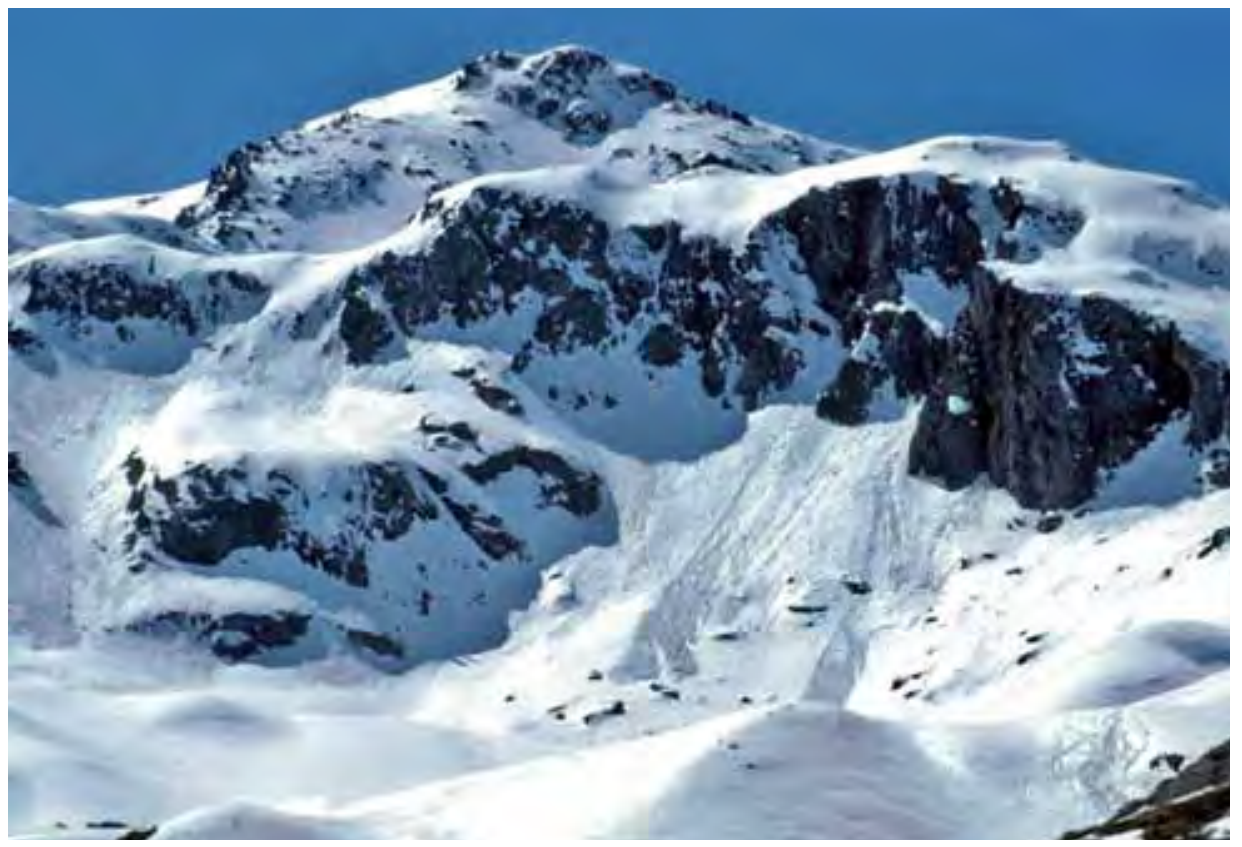

Figura 6. Aludes de nieve suelta húmeda en las laderas del circo de Espelunciecha (zona de control 2).



Figura 7. Laderas innivadas en el sector de El Portalet (zona de control 1). 


\section{Conclusiones}

La elaboración de cartografías de zonas probables de salida de aludes a partir del empleo de Sistemas de Información Geográfica es una ayuda importante en la caracterización espacial de este tipo de fenómenos, muy presentes durante la temporada invernal en la alta montaña aragonesa.

En el presente trabajo se ha realizado, en el ámbito del alto valle del río Gállego, una doble modelización a escala espacial de trabajo 1:25.000, teniendo presente la evolución a nivel mensual de factores condicionantes básicos (altitud de la isoterma de $0^{\circ} \mathrm{C}$, pendientes y cubierta del terreno) (modelo MGZPSA) y el papel adicional de la radiación solar (modelo MRSZPSA).

Los resultados parecen confirmar la validez de ambos modelos, y permiten establecer la importancia del factor radiación solar a la hora de matizar, a lo largo del invierno, la distribución espacial precisa de los sectores de arranque de avalanchas. Suponen, en cualquier caso, una primera aproximación que habrá de confirmarse en el futuro incrementando las zonas de control y el número de eventos observados.

\section{Agradecimientos}

Investigación subvencionada por el Ministerio de Ciencia e Innovación (Proyecto CGL2007-62614/BTE; "Reconstrucción de episodios de aludes a partir del análisis dendrocronológico en los Pirineos").

\section{Referencias bibliográficas}

Chueca, J., Julián, A. (2004). Caracterización y tipología de canales de aludes en el valle de Ordesa (Pirineo central español). Bol. R. Soc. Esp. Hist. Nat. (Sec. Geol.), 99 (1-4): 93-103.

Chueca, J., Julián, A., Peña, J. L., Espinalt, M. (2000). Mapa geomorfológico del Alto Gállego (Pirineo aragonés). Boletín Glaciológico Aragonés, 1.

Chueca, J., Julián, A., Palomo, M., Muntán, E., Oller, P., Barriendos, M., GutiéRREZ, E. (2009). Factores geomorfológicos y nivometeorológicos condicionantes de aludes en el circo de Musales (Pirineo central aragonés): el evento de abril de 2008. Actas II Congreso Ibérico de la International Permafrost Association, 39-40.

De la Riva, J. (2000). Caracterización climática del alto valle de Tena. Boletín Glaciológico Aragonés, 1: 81-109.

Ibarra, P., Pérez Cabello, F. (2000). La vegetación del alto valle de Tena. Boletín Glaciológico Aragonés, 1: 127-149. 
Julián, A., Chueca, J. (1999). Cartografía de zonas probables de aludes en el valle de Ordesa (Pirineo aragonés). Geographicalia, 37: 73-86.

Julián, A., Chueca, J. (2008). Cartografía de zonas de salida de aludes en el valle de Ordesa (Pirineo central español): factores condicionantes y evolución temporal. En Benavente, J. y Gracia, F.J. (Eds.): Trabajos de Geomorfología en España, 20062008. SEG, Cádiz, 413-416.

Julián, A., Chueca, J., Peña, J. L. (2000). El relieve del alto Gállego (Pirineo aragonés). Boletín Glaciológico Aragonés, 1: 45-79.

Julián, A., Peña, J. L., Chueca, J., Zabalza, J., Lapeña, A., LóPez, J. I. (2001). Cartografía de zonas probables de aludes en el Pirineo aragonés: metodología y resultados. Boletín de la A.G.E., 30: 119-134.

LANDIS, J. R., Koch, G. G. (1977). The measurement of observer agreement for categorical data. Biometrics, 33: 159-174.

López Guarga, R., Sarasa, A., Oller, P. (1997). Caracterización, simulación y prevención de aludes en el barranco de Secras. Túnel de Somport (Huesca). IV Simposio Nacional sobre taludes y laderas inestables, Granada, 703-717.

McClung, D., Schaerer, P. (1996). Avalanchas. Ediciones Desnivel, Madrid, 309 pp.

Palomo, M. (2007). Los aludes en el Circo de Piedrafita (Pirineo aragonés): el evento del 8 de febrero de 1996. Boletín Glaciológico Aragonés, 8: 61-83.

Palomo, M., Julián, A., Chueca, J. (2008). Aplicación de un análisis multicriterio a la delimitación de zonas probables de aludes (valle del Aguas Limpias, Pirineo central español). En Benavente, J. y Gracia, F.J. (Eds.): Trabajos de Geomorfología en España, 2006-2008. Sociedad Española de Geomorfología, Cádiz, 417-420. 